\title{
Construction des surfaces analytiques ayant une variété double ordinaire donnée
}

\author{
Par \\ Hidekazu ONISHI \\ (Reçu le 26 décembre, 1964)
}

\section{Introduction.}

Nous avons démontré dans le mémoire [2], n $\mathrm{n}^{0} 9$, qu'un domaine $\Delta$ intérieurement ramifié sur un polycylindre $\underline{\Delta}$ à l'espace de $n$ variables complexes $\left(x_{1}, \cdots, x_{n}\right)$ peut se représenter par une surface analytique $\Sigma$ de type $(K)^{1)}$, n'admettant pour sa variété singulière $^{1)}$ de dimension $n-1$ qu'une variété double ordinaire $^{1)} T$.

La variété double ordinaire $T$ d'une surface analytique $\Sigma$ de type $(K)$ joue un rôle essentiel dans l'étude sur le domaine intérieurement ramifié $\Delta$. En fait, le théorème $6,[3]$, montre que le problème $(A)$ est ou bien affirmatif pour tous les domaines $\Delta$ qui se représentent par des surfaces $\Sigma$ de type $(K)$, ayant la même variété double ordinaire donnée $T$, ou bien toujours négatif; autrement dit, la réponse au problème $(A)$ ne dépend que de la variété double ordinaire $T$.

Dans ce mémoire, nous nous proposons réciproquement de montrer l'existence d'une surface $\Sigma$ (et donc, l'existence d'un domaine intérieurement ramifié sur $\underline{\Delta}$ ), de type $(K)$, ayant une variété double ordinaire donnée $T$. (Voir le théorème $3, \mathrm{n}^{0}{ }^{6}$.)

Le théorème peut s'étendre au cas où est indiqué un nombre fini de variétés multiples ordinaires $T_{i}(i=1, \cdots, m)$ d'ordres quelconques $q_{i}$. (Voir le théorème 3 bis, $\mathrm{n}^{0} 11$.)

Les résultats seront utilisés dans le mémoire qui suivra, pour

1) Pour définitions et terminologies, voir nos 1 et 2 du présent mémoire. 
obtenir un critère concret du problème $(A)$ et construire quelques exemples de domaines intérieurement ramifiés.

\section{$\S 1$. Préliminaires.}

1. Soit $\mathscr{D}$ un domaine univalent quelconque (fini ou non) dans l'espace de $n$ variables complexes $\left(x_{1}, \cdots, x_{n}\right)$, et soit $\mathscr{D}$ un domaine (connexe ou non) intérieurement ramifié sur $\mathscr{D}$, à $\nu$ feuillets.

Etant donnée une fonction $\eta(P)$ holomorphe sur $\mathscr{D}$, on dit que $\eta(P)$ est une fonction propre sur $\mathscr{D}$, si les éléments de fonction de $r(P)$ aux différents points de $\mathscr{D}$ situés sur un même point de $\mathscr{D}$ sont toujours différents.

Considérons, à l'espace de $n+1$ variables $((x), y)$, une surface analytique $\Sigma$ :

$$
y=\eta(P) \quad(P \in \mathscr{D}),
$$

$\eta(P)$ étant une fonction propre sur $\mathscr{D} . \quad \Sigma$ est contenue dans un domaine univalent $\mathcal{L}^{*}$ de la forme:

$$
(x) \in \underline{\mathscr{D}}, \quad|y|<+\infty .
$$

Dans ce qui suit, le domaine (ou l'ensemble, en général) $\mathscr{L}^{*}$ de cette forme sera noté $\mathscr{D}^{*}=(\mathscr{D}, Y), Y$ désignant le plan de la variable $y$.

Tout point $P$ de $\mathscr{D}$, sauf peut-être ceux appartenant à une variété $^{2)} \tau$ à $n-1$ dimensions dans $\mathscr{D}$, correspond biunivoquement au point $(\pi(P), \eta(P))$ de $\Sigma, \pi$ designant l'opération de projection de $\mathscr{D}$ sur $\mathscr{D}$. L'image de $\tau$ sur $\Sigma$ sera noté $T$.

Notons $\sigma$ la surface critique de $\mathscr{D}$, et posons

$$
\sigma=\pi(\sigma), \underline{\tau}=\pi(\tau), \hat{\tau}=\pi^{-1}(\tau) .
$$

Si l'on forme, pour les points $(x)$ de $\underline{D}-\underline{\sigma}$, le produit

$$
\Phi((x), y)=\prod_{j=1}^{v}\left[y-\eta\left(P_{j}\right)\right] \quad\left(P_{j} \in \pi^{-1}(x)\right),
$$

2) K. Oka, [1], n" 7 . 
$\Phi((x), y)$ devient un pseudo-polynôme de degré $\nu$ en $y$, à coefficients holomorphes dans $\mathscr{D}$; et la surface $\Sigma$ peut s'éxprimer dans $\mathscr{D}^{*}$ par l'équation

$$
\Phi((x), y)=0 .
$$

Comme $\gamma(P)$ est propre sur $\mathscr{D}, \Phi((x), y)$ n'admet aucun facteur multiple. Donc, la variété singulière de $\Sigma$ est donnée par les équations

$$
\Phi=0, \frac{\partial \Phi}{\partial x_{j}}=0(j=1, \cdots, n) \text { et } \frac{\partial \Phi}{\partial y}=0 .
$$

2. Etant donnée une fonction $\eta(P)$ holomorphe et propre sur $\mathscr{D}$, supposons que la surface $\sum: y=\eta(P)$ satisfasse aux conditions suivantes:

$1^{\circ}$ en dehors de $T, \Sigma$ n'admet aucune variété singulière de dimension $n-1$;

$2^{\circ} \quad \underline{\tau}$ et $\underline{\sigma}$ n'ont aucune composante commune;

$3^{\circ} \tau$ est un revêtement (intérieurement ramifié) de $\tau$, à deux feuillets; autrement dit, l'image $\hat{T}$ de $\hat{\tau}$ sur $\Sigma$ est un revêtement (intérieurement ramifié) de $\tau$, à $\nu-1$ feuillets;

$4^{\circ}$ par tout point $M$ de $T$, sauf les points $M$ appartenant à une sous-variété $S$ de dimension au plus $n-2$ sur $T$, passent deux et seulement deux feuillets univalents ${ }^{3)}$ de $\sum$, avec plans tangents (à $n$ dimensions) distincts en $M$.

Nous dirons dans ce cas que la surface $\Sigma$ est de type $(K)$ sur $\mathscr{D}$ [ou que la fonction $\eta(P)$ possède la propriété $(K)$ dans $\mathscr{D}$ ]. Tout point $M$ de $T-S$ sera appelé point double ordinaire de $\Sigma$, et $T$ variété double ordinaire ${ }^{4)}$ de $\Sigma$.

\section{§ 2. Variétés singulières de certaines surfaces analytiques.}

3. Soit à nouveau $\mathscr{D}$ un domaine univalent quelconque (fini ou non) dans l'espace de $n+1$ variables complexes $\left(x_{1}, \cdots, x_{n+1}\right)$. Considérons, à l'espace de $n+2$ variables complexes $((x), t)$, un domaine univalent $\tilde{\mathscr{D}}$ de la forme:

3) On tient compte des conditions $2^{\circ}$ et $3^{\circ}$.

4) $\Sigma$ peut naturellement avoir sur $S$, des singularités plus compliquées que les points doubles ordinaires. 


$$
(x) \in \mathscr{D}, \quad|t|<r,
$$

où $r$ est un nombre positif quelconque ou $r=+\infty$.

Soit $F((x), t)$ une fonction holomorphe (et non identiquement nulle) dans $\tilde{\mathscr{D}}$. Supposons que $F((x), t)$ satisfasse aux deux conditions suivantes:

$1^{\circ}$ en tout point de $\tilde{D}$, la décomposition de $F((x), t)$ en produit de facteurs irréductibles n'admet aucun facteur multiple;

$2^{\circ}$ pour toute valeur $c(|c|<r)$ de la variable $t$, la fonction $F((x), c)$ de variables $(x)$ s'annule au moins en un point de $\mathscr{D}$.

L'ensemble $\Sigma_{c}: F((x), c)=0$ est ou bien une surface analytique de dimension $n$ dans $\mathscr{D}$, ou bien il coincide avec tout le domaine $\mathscr{D}^{5)}$. D'ailleurs, il peut se faire que pour certaines valeurs de $c, \sum_{c}$ contienne des composantes le long desquelles les ordres de zéros de $F((x), c)$ sont plus grands qu'un. ${ }^{6)}$

Lorsque $\sum_{c}$ est une surface de dimension $n$, désignons par $\tau$ la variété singulière de $\sum_{c} . \tau_{c}$ est donnée par les équations

$$
F((x), c)=0, \frac{\partial F}{\partial x_{j}}((x), c)=0 \quad(j=1, \cdots, n+1),
$$

si et seulement si l'ordre de zéros de $F((x), c)$ est premier.

On obtient le théorème suivant:

Théorème 1. Soit $F((x), t)$ une fonction holomorphe dans $\tilde{D}$ satisfaisant aux deux conditions expliquées plus haut. Pour toute valeur de $c$ telle que $|c|<r$, sauf un nombre dénombrable de valeurs de $c$,

$1^{\circ} \sum_{c}: F((x), c)=0$ est une surface analytique (connexe ou non) à $n$ dimensions dans $\mathscr{D}$;

$2^{\circ} \quad$ l'ordre de zéros de $F((x), c)$ est premier;

$3^{\circ}$ la variété singulière $\tau_{c}$ de $\sum_{c}$ s'obtient par les équations

$$
F(x), c)=0, \frac{\partial F}{\partial x_{j}}((x), c)=0 \quad(j=1, \cdots, n+1) \text {, et } \frac{\partial F}{\partial t}((x), c)=0^{7)} \text {. }
$$

5) Par exemple, si $F((x), t)=t x_{1}, F((x), c)$ s'annule identiquement pour $c=0$.

6) Par exemple, si $F((x), t)=x_{1}{ }^{2}+t x_{2}$, on a $F((x), 0)=x_{1}{ }^{2}$. 
Notons que les premiers deux énoncés du théorème entraînent que la variété singulière $\tau_{c}$ de $\sum_{c}$ s'obtient par

$$
F((x), c)=0, \quad \frac{\partial F}{\partial x_{j}}((x), c)=0 \quad(j=1, \cdots, n+1) .
$$

Pour mettre le dernier énoncé du théorème en évidence, envisageons dans $\tilde{\mathscr{D}}$ la surface analytique (à $n+1$ dimensions) $\tilde{\Sigma}$ définie par l'équation

$$
F((x), t)=0 .
$$

Soit $\tilde{\tau}$ la variété singulière de $\tilde{\Sigma}$. Par l'hypothèse posée sur la fonction $F((x), t)$, $\tilde{\tau}$ est donnée par les équations

$$
F=0, \frac{\partial F}{\partial x_{j}}=0 \quad(j=1, \cdots, n+1), \text { et } \frac{\partial F}{\partial t}=0 .
$$

Pour une valeur quelconque $c(|c|<r)$ de $t$, on désigne par $\tilde{\tau}_{c}$ l'intersection de $\tilde{\tau}$ et du plan (à $n+1$ dimensions dans $\tilde{\mathscr{D}}$ ) $\Pi_{\mathrm{c}}: t=c$.

Cela posé, le dernier énoncé du théorème équivaut à dire que la variété singulière $\tau_{c}$ de $\sum_{c}$ coincide avec la projection (sur $\mathscr{D}$ ) $\pi_{c}\left(\tilde{\tau}_{c}\right)$ de $\tilde{\tau}_{c}:$

$$
\tau_{c}=\pi_{c}\left(\tilde{\tau}_{c}\right)^{8)} .
$$

4. Pour démontrer le théorème, considérons dans $\mathscr{D}$ l'ensemble anlytique $S$ :

$$
F((x), t)=0, \frac{\partial F}{\partial x_{j}}((x), t)=0 \quad(j=1, \cdots, n+1) .
$$

Si $S$ est vide, les énoncés du théorème sont trivialement vrais pour toute valeur de $c(|c|<r)$.

Soit alors $S^{0}$ une composante de $S$, et $m$ sa dimension; on a

7) Pour certaines valeurs de $c$, il arrive que certains points de $\tau_{c}$, ne satisfassent pas à $\frac{\partial F}{\partial t}((x), c)=0$. Par exemple, si $F((x), t)=x_{1}{ }^{2}+x_{2}{ }^{2}+t x_{3}$, on obtient d'une part $\tau_{c}=\left\{\left(0,0, x_{3}\right)\right\}$ pour $c=0$, et d'autre part $\frac{\partial F}{\partial t}((x), 0)=x_{3}$.

8) On a évidemment $\pi_{c}\left(\tilde{\tau}_{c}\right) \subset \tau_{c}$. Il peut se faire qu'on ait $\pi_{c}\left(\tilde{\tau}_{c}\right) \neq \tau_{c}$, comme on le voit dans la note 7 ). 
$0 \leqq m \leqq n+1^{9)}$. Soit $M$ un point régulier quelconque de $S^{0}$. Dans un voisinage convenable $V$ de $M, S^{0} \cap V$ peut se représenter, au moyens de $m$ paramètres complexes $\left(u_{1}, \cdots, u_{m}\right)$, par les formules:

$$
x_{j}=\xi_{j}(u) \quad(j=1, \cdots, n+1), \quad t=\theta(u),
$$

où $\xi_{j}(u)$ et $\theta(u)$ sont des fonctions holomorphes au voisinage $U$ de l'origine de l'espace $\left(u_{1}, \cdots, u_{m}\right)$. On a identiquement dans $U$

$$
F((\xi), \theta)=0, \frac{\partial F}{\partial x_{j}}((\xi), \theta)=0 \quad(j=1, \cdots, n+1) .
$$

En différentiant par $u_{k}$ les deux membres de la relation $F((\xi), \theta)=0$, on a identiquement dans $U$

$$
\sum_{j=1}^{n+1} \frac{\partial F}{\partial x_{j}}((\xi), \theta) \frac{\partial \xi_{j}}{\partial u_{k}}+\frac{\partial F}{\partial t}((\xi), \theta) \frac{\partial \theta}{\partial u_{k}}=0 .
$$

Par suite, on a identiquement dans $U$

$$
\frac{\partial F}{\partial t}((\xi), \theta) \frac{\partial \theta}{\partial u_{k}}=0 \quad(k=1, \cdots, m) .
$$

Il y a deux cas suivants :

Cas $1^{\circ}$. Si l'on a identiquement dans $U$

$$
\frac{\partial \theta}{\partial u_{k}}=0 \quad(k=1, \cdots, m),
$$

la fonction $\theta(u)$ est égale à une constante $c_{0}$. Ceci signifie que la composante $S^{0}$ est contenue dans le plan $\Pi_{c_{0}}: t=c_{0}$; nous dirons dans ce cas que la composante $S^{0}$ est de première espèce. ${ }^{107}$

Cas $2^{\circ}$. Si, au contraire, $S^{0}$ n'est pas de première espèce, nous dirons que $S^{0}$ est de seconde espèce.

Si $S^{0}$ est de seconde espèce, on a identiquement dans $U$

$$
\frac{\partial \mathrm{F}}{\partial t}((\xi), \theta)=0
$$

9) La dimension $m$ de $S^{\theta}$ peut atteindre à $n+1$, comme on le voit dans l'exemple: $F\left(x_{1}, \cdots, x_{n+1}, t\right)=t x_{1}$.

10) Lorsque $m=0, S^{\ominus}$ est $a$ priori de première espèce. 
c'est-à-dire, on a identiquement sur $S^{0}$

$$
\frac{\partial F}{\partial t}=0
$$

et donc, $S^{0}$ est contenue dans la variété singulière $\tilde{\tau}$ de $\tilde{\Sigma}$ : $F((x), t)=0$.

De là, on voit que, si $S^{0}$ est de seconde espèce, sa dimension $m$ est au plus $n$. De plus, l'intersection $S_{c}^{0}$ de $S^{0}$ (de seconde espèce) et du plan $\Pi_{c}: t=c$ est de dimension au plus $n-1$, puisque, par définition, la composante $S^{0}$ de seconde espèce n'est contenue dans aucun plan $\Pi_{c}$.

Maintenant, notons $S^{(1)}, S^{(2)}, \ldots$ les composantes de première espèce de $S$; et $S^{*}$ la réunion des composantes de seconde espèce de $S$; chaque $S^{(i)}$ est contenue dans un plan $t=c_{i}$ $(i=1,2, \cdots)$.

Désignons, pour toute valeur de $c(|c|<r)$, par $S_{c}$ [resp. $S_{c}^{*}$ ] l'intersection de $S$ [resp. $S^{*}$ ] et du plan $\Pi_{c}$; on a $S_{c}{ }^{*} \subset S_{c}$. Par définition, la projection $\pi_{c}\left(S_{c}\right)$ de $S_{c}$ sur $\mathscr{D}$ s'exprime par

$$
\left(\pi_{c}\left(S_{c}\right)\right) \quad F((x), c)=0, \frac{\partial F}{\partial x_{j}}((x), c)=0 \quad(j=1, \cdots, n+1) .
$$

Si $c(|c|<r)$ est une valeur de $t$, différente de $c_{i}(i=1,2, \cdots)$, il est évident qu'on a

$$
S_{c}=S_{c}^{*},
$$

puisque le plan $\Pi_{c}\left(c \neq c_{i}\right)$ ne contient pas de point appartenant à aucune composante de première espèce de $S$.

Donc, il résulte de ce que nous venons de voir, que:

a) $S_{c}\left(c \neq c_{i}\right)$, et donc $\pi_{c}\left(S_{c}\right)$ est de dimension au plus $n-1$, et que

b) on a $S_{c} \subset \tilde{\tau}_{c}$, et donc $\pi_{c}\left(S_{c}\right) \subset \pi_{c}\left(\tilde{\tau}_{c}\right)$.

D'ailleurs, l'énoncé $a$ ) entraîne aussitôt que:

$1^{\circ} F((x), c)\left(c \neq c_{i}\right)$ ne s'annule pas identiquement dans $\mathscr{D}$, et que $2^{\circ} \quad$ l'ordre de zéros de $F((x), c)$ est premier;

et ceci montre que la variété singulière $\tau_{c}$ de $\Sigma_{c}$ est identique à $\pi_{c}\left(S_{c}\right)$. (On tient compte des équations ci-dessus de $\pi_{c}\left(S_{c}\right)$.) 
Donc, l'énoncé $b$ ) entraîne qu'on a

$$
\tau_{c} \subset \pi_{c}\left(\tilde{\tau}_{c}\right)
$$

et donc, on a, pour $c \neq c_{i}(i=1,2, \cdots)$

$$
\tau_{c}=\pi_{c}\left(\tilde{\tau}_{c}\right)^{11)} .
$$

Ceci achève la démonstration du théorème 1 .

5. Comme un corollaire du théorème 1 , on a le théorème suivant:

Théorème 2. Soit $\Psi(x)$ une fonction holomorphe (et non identiquement nulle) dans $\mathscr{D}$, qui s'annule au moins en un point de $\mathscr{D}$, et qui n'admet pas de facteur multiple en aucun point de $\mathscr{D}$. Soit $\Phi(x)$ une fonction quelconque holomorphe dans $\mathscr{D}$, et $p$ un entier quelconque tel que $p \geqq 2$.

On peut trouver un nombre positif $r$ tel que, pour toute valeur de c telle que $|c|<r$, sauf un nombre dénombrable de valeurs de $c$,

$1^{\circ}$ l'équation

$$
\Psi(x)+c[\Phi(x)]^{p}=0
$$

définisse dans $\mathscr{D}$ une surface analytique (à $n$ dimensions) $\sum_{c}$, l'ordre de zéros de $\Psi+c \Phi^{p}$ étant premier;

$2^{\circ}$ la variété singulière $\tau_{c}$ de $\Sigma_{c} s^{\prime}$ obtienne par les équations qui ne dépendent pas de $c$,

$$
\Phi=0, \Psi=0, \frac{\partial \Psi}{\partial x_{j}}=0 \quad(j=1, \cdots, n+1) ;
$$

en d'autres termes, $\tau_{c}$ soit identique à l'intersection $\tau^{*}$ (qui ne dépend pas de c) de la surface $T: \Phi=0$ et de la variété singulière $\tau_{0}$ de la surface $\Sigma_{0}: \Psi=0$; et que

$3^{\circ}$ si un point $\left(x^{0}\right)$ de $\sum_{c}$ satisfaisant $\grave{a} \Phi=0$ et $\grave{a} \Psi=0$ est un point multiple ordinaire d'ordre $\left.q(q \geqq 1)^{12}\right)$ de la surface $\Sigma_{0}$, et si $q<p,\left(x^{0}\right)$ soit encore un point multiple ordinaire d'ordre $q$ de la surface $\Sigma_{c}$, de manière que les $q$ feuillets (univalents) de $\Sigma_{0}$

11) Voir la note 8) du bas de la page 287.

12) Le point simple correspond au cas où $q=1$, et le point double ordinaire correspond au cas où $q=2$, etc. 
passant par $\left(x^{0}\right)$ et ceux de $\sum_{c}$ aient tous les $q$ plans tangents en commun en $\left(x^{0}\right)$.

Pour vérifier le théorème, posons

$$
F((x), t)=\Psi(x)+t[\Phi(x)]^{p},
$$

$t$ étant une nouvelle variable complexe.

On peut aisément trouver un nombre positif $r$ tel qu'il satisfasse à la deuxième condition de l'hypothèse du théorème 1 , posée sur $F((x), t)$ (voir $\mathrm{n}^{0} 3$ ). Le premier énoncé du théorème 2 est alors évident d'après le théorème 1 .

Le deuxième énoncé est aussi immédiat, puisque, d'après le théorème $1, \tau_{c}$ s'obtient par les équations

$$
\begin{gathered}
F((x), c)=\Psi+c \Phi^{p}=0, \frac{\partial F}{\partial x_{j}}((x), c)=\frac{\partial \Psi}{\partial x_{j}}+c p \Phi^{p-1} \frac{\partial \Phi}{\partial x_{j}}=0(j=1, \cdots, n+1), \\
\text { et } \frac{\partial F}{\partial t}((x), c)=\Phi^{p}=0,
\end{gathered}
$$

où l'on a $p-1 \geqq 1$.

Quant au dernier énoncé, la partie homogène de plus petit degré du développement de Taylor de la fonction $\Psi+c \Phi^{p}$ au point $\left(x^{0}\right)$ est identique à celle de degré $q(<p)$ renfermée dans le développement de $\Psi$, et qui décompose, par hypothèse, en $q$ facteurs linéaires distincts; d'où l'énoncé. $\quad$ c. q. f. d.

Remarque. Si $\Psi(x)$ admet des facteurs multiples, mais si $\Psi(x)$ et $\Phi(x)$ n'admettent aucun facteur commun, les énoncés $1^{\circ}$ et $2^{\circ}$ du théorème 2 sont encore vrais, d'après le théorème 1 ; et la variété singulière $\tau_{c}$ de $\sum_{c}$ est contenue dans l'intersection des deux surfaces $\Phi=0$ et $\Psi=0$.

Par exemple: on peut trouver un nombre positif $r$ tel que, pour toute valeur de $c$ telle que $|c|<r$, sauf un nombre dénombrable de valeurs de $c$, l'équation

$$
\Psi(x)=c
$$

définisse une surface régulière $\Sigma_{c}$ dans $\mathscr{D}$, l'ordre de zéros de $\Psi(x)-c$ étant premier. 


\section{$\S 3$. Construction des surfaces ayant une variété double ordinaire donnée.}

6. Soit $\mathscr{D}^{0}$ un domaine univalent quelconque (fini ou non) dans l'espace de $n-1$ variables complexes $\left(x_{1}, \cdots, x_{n-1}\right)$, et soit $\mathscr{D}^{0}$ un domaine intérieurement ramifié sur $\mathscr{D}^{0}$, à $\nu$ feuillets. La surface critique de $\mathscr{D}^{0}$ sera désignée par $\sigma^{0}$.

Supposons données deux fonctions $\eta(Q), \zeta(Q)$ holomorphes sur $\mathscr{D}^{0}$, dont $\eta(Q)$ est supposée propre sur $\mathscr{D}^{0}$.

Soit, à l'espace de $n$ variables complexes $((x), y), \underline{T}$ une surface analytique définie par

$$
y=\eta(Q) \quad\left(Q \in \mathscr{D}^{0}\right)
$$

$\underline{T}$ est contenue dans un domaine univalent $\underline{\mathscr{D}}=\left(\mathscr{D}^{0}, Y\right)[Y$ désignant le plan de la variable $y]$ :

$$
(x) \in \underline{D}^{0}, \quad|y|<+\infty .
$$

Considérons, à l'espace de $n+1$ variables complexes $((x), y, z)$, une variété analytique (à $n-1$ dimensions) $T$ :

$$
y=r(Q), \quad z=\zeta(Q) \quad\left(Q \in \mathscr{D}^{0}\right) ;
$$

$T$ est contenue dans un domaine univalent $\Delta=\left(\mathscr{D}^{0}, Y, Z\right)=(\mathscr{D}, Z)$ [ $Z$ désignant le plan de la variable $z]$ :

$$
(x) \in \underline{\mathscr{D}}^{0}, \quad|y|<+\infty, \quad|z|<+\infty .
$$

Nous allons établir le théorème suivant:

Théorème $3{ }^{13)}$ Etant donnée à l'espace de $n+1(n \geqq 1)$ variables complexes, une variété analytique $T \subset \Delta$ à $n-1$ dimensions de la forme expliquée ci-dessus, il existe dans $\Delta$ une surface analytique $\Sigma$ de dimension $n$, ayant $T$ pour sa variéte double ordinaire, sans avoir aucune variété singulière de dimension $n-1$ en dehors

13) Le théorème s'applique même au cas où $n=1$. Dans ce cas, $\mathscr{D}^{\ominus}$ se compose d'un seul point, et $\mathscr{D}^{0}$ de $\nu$ points distincts; $T$ est donnée comme $\nu$ points $\left(\eta_{j}, \zeta_{j}\right)\left(\eta_{j} \neq \eta_{k}\right.$ pour $\left.j \neq k\right)$ sur le plan $(y, z)$. $\sum$ s'obtient comme une courbe sur le plan $(y, z)$ ayant les $\nu$ points de $T$ pour ses points doubles ordinaires, sans singularité d'ailleurs. 
de $T$, et de plus, sans aucune singularité en dehors de $\hat{T}=\pi^{-1}(\underline{T})$, $\pi$ désignant l'opération de projection de $\Sigma$ sur $\mathscr{D}$. En outre, on peut construire $\Sigma$ de manière qu'elle admette $2 \nu$ feuillets sur $\underline{D}$, et qu'elle soit de type $(K)$ sur $\mathscr{D}$.

Ce théorème peut s'étendre au cas où est indiqué un nombre fini de variétés multiples ordinaires $T_{i}$ d'ordres quelconques $q_{i}$ (voir $\mathrm{n}^{0} 11$, théorème 3 bis).

7. Démontrons le théorème 3. Lorsqu'on a $\nu=1$, le théorème est évident. Dans ce cas, en effet, la surface voulue $\Sigma$ s'obtient par une équation du second degré en $z$ :

$$
[z-\zeta(x)]^{2}-[y-\eta(x)]^{2}=0 .
$$

Il suffit donc de démontrer le théorème aux cas où $\nu \geqq 2$.

Cela étant, soit $\underline{Q}=(x)$ un point quelconque de $\mathscr{D}^{0}$ qui n'appartient pas à la projection $\sigma^{0}$ de la surface critique $\sigma^{0}$ de $\mathscr{D}^{0}$. On désigne par $Q_{1}, \cdots, Q_{\nu}$ les $\nu(\nu \geqq 2)$ points distincts de $\mathscr{D}^{0}$ situés sur le même point $\underline{Q}$.

On aura besoin du lemme suivant:

Lemme. Si $\nu \geqq 2$, on peut trouver deux nombres complexes distincts $\alpha_{1}, \alpha_{2}$ de façon que l'on ait

$$
\zeta\left(Q_{j}\right)+\alpha_{1}\left[\eta\left(Q_{l}\right)-\eta\left(Q_{j}\right)\right] \neq \zeta\left(Q_{k}\right)+\alpha_{2}\left[\eta\left(Q_{l}\right)-\eta\left(Q_{k}\right)\right],
$$

pour tout point $\underline{Q}$ de $\underline{D}^{0}$, sauf peut-être $\underline{Q}$ appartenant à une surface analytique $E^{0}\left(\supset \sigma^{0}\right)$ de codimension un dans $\mathscr{D}^{0}$, et pour tout système d'indices $(j, k, l)(j, k, l=1, \cdots, \nu)$, sauf les $\nu$ systèmes où l'on a $j=k=l$.

Il est clair, par la méthode de prolongement analytique, que pour verifier le lemme, il suffit de trouver deux nombres complexes distincts $\alpha_{1}, \alpha_{2}$ tels que l'on ait, pour un seul point $\underline{Q}^{0}$ de $\underline{\mathcal{D}^{0}}$,

$$
\begin{gathered}
\zeta\left(Q_{j}^{0}\right)+\alpha_{1}\left[\eta\left(Q_{l}^{0}\right)-r\left(Q_{j}^{0}\right)\right] \neq \zeta\left(Q_{k}^{0}\right)+\alpha_{2}\left[\eta\left(Q_{l}^{0}\right)-\eta\left(Q_{k}^{0}\right)\right] \\
(j, k, l=1, \cdots, \nu ; j \neq l \text { ou } k \neq l) .
\end{gathered}
$$

Mais, ceci est immédiat, puicque, $r(Q)$ étant propre sur $\mathscr{D}^{0}$, on peut d'abord trouver un point $\underline{Q}^{0}$ de $\underline{D}^{0}-\underline{\sigma}^{0}$ tel que l'on ait 


$$
\eta\left(Q_{k}^{0}\right)-\eta\left(Q_{l}^{0}\right) \neq 0 \quad(k, l=1, \cdots, \nu ; k \neq l)
$$

et qu'on peut ensuite choisir un point sur le plan $\left(\alpha_{1}, \alpha_{2}\right)$ de dimension complexe deux, en évitant ${ }^{14)}$ un nombre fini de droites de dimension complexe un.

c. q. f. d.

Remarque. Parmi les inégalités dans l'énoncé du lemme, se comptent quelques inégalités particulières correspondant aux trois cas où l'on a $j=k$ ou $j=l$ ou $k=l$.

Lorsqu'on a $j=k(j \neq l)$, on a facilement

$$
\eta\left(Q_{k}\right)-\eta\left(Q_{l}\right) \neq 0 \quad\left(k \neq l ; \underline{Q} \in \underline{D}^{0}-E^{0}\right) ;
$$

et lorsqu'on a $k=l(k \neq j)$ [resp. $j=l(j \neq k)]$, on a

$$
\zeta\left(Q_{j}\right)-\alpha_{i} \eta\left(Q_{j}\right) \neq \zeta\left(Q_{k}\right)-\alpha_{i} \eta\left(Q_{k}\right) \quad\left(j \neq k ; i=1,2 ; \underline{Q} \in \underline{D}^{0}-E^{0}\right) .
$$

8. Considérons un domaine (à $n$ dimensions) $\mathscr{D}$ qui est obtenu comme le produit du domaine $\mathscr{D}^{0}$ (intérieurement ramifié sur $\left.\underline{D}^{0}\right)$ et du plan $Y$ de la variable $y$ :

$$
\mathscr{D}=\left(\mathscr{D}^{0}, Y\right) \text {; }
$$

$\mathscr{D}$ est un domaine intérieurement ramifié sur $\mathscr{D}=\left(\mathscr{D}^{0}, Y\right)$, à $\nu$ feuillets, et ayant $\sigma=\left(\sigma^{0}, Y\right)$ pour sa surface critique. En désignant par $\underline{\sigma}$ la projection de $\sigma$ sur $\underline{\mathscr{D}}$, on a

$$
\underline{\sigma}=\left(\underline{\sigma}^{0}, Y\right) ;
$$

et $\underline{\sigma}$ est contenue dans la surface analytique (à $n-1$ dimensions) $E=\left(E^{0}, Y\right), E^{0}$ étant la surface mentionnée au lemme du $\mathrm{n}^{\mathrm{o}}$ précédent.

Il est clair que l'intersection $\underline{S}$ de $E$ et de $T: y=\eta(Q)$ est une variété de dimension $n-2$.

Maintenant, pour $i=1,2$, posons sur $\mathscr{D}=\left(\mathscr{D}^{0}, Y\right)$

$$
\zeta_{i}(P)=\zeta(Q)+\alpha_{i}[y-r(Q)] \quad\left(P=(Q, y) \in \mathscr{D}, Q \in \mathscr{D}^{0}\right),
$$

$\alpha_{i}$ étant les deux nombres complexes mentionnés au lemme du $\mathrm{n}^{0}$ précédent. $\zeta_{i}(P)$ sont des fonctions holomorphes sur $\mathscr{D}$.

14) La condition: $\alpha_{1} \neq \alpha_{2}$ est aussi comptée parmi ces inégalités comme un cas particulier où l'on a $j=k(k \neq l)$. 
Envisageons les valeurs de ces fonctions prises sur $\underline{T}: y$ $=\eta(Q)$. Pour cela, prenons un point quelconque $\underline{P}$ de $\underline{T}$ n'appartenant pas à $\underline{S}$. Soient $\underline{P}=\left(\underline{Q}, \eta\left(Q_{l}\right)\right)$ ses coordonnées, $\underline{Q}$ étant un point de $\mathscr{D}^{0}-E^{0}\left(E^{0} \supset \underline{\sigma}^{0}\right)$, et $Q_{l}$ un des points $Q_{1}, \cdots, Q_{\nu}$ de $\mathscr{D}^{0}$ situés sur $\underline{Q}$.

Sur le point $\underline{P}$, se situent alors $\nu$ points distincts $P_{j}(j=1, \cdots$,

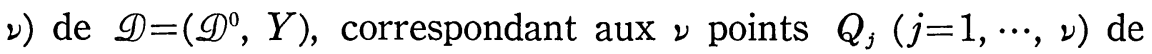
$\mathscr{D}^{0}$ :

$$
P_{j}=\left(Q_{j}, \eta\left(Q_{l}\right)\right) \quad(j=1, \cdots, \nu ; \underline{P} \in \underline{T}-\underline{S}) .
$$

On a, pour $i=1,2$,

$$
\zeta_{i}\left(P_{j}\right)=\zeta\left(Q_{j}\right)+\alpha_{i}\left[\eta\left(Q_{l}\right)-\eta\left(Q_{j}\right)\right](j=1, \cdots, \nu) .
$$

D'une part, on obtient, pour le point $P_{l}=\left(Q_{l}, \eta\left(Q_{l}\right)\right)$,

$$
\zeta_{1}\left(P_{l}\right)=\zeta_{2}\left(P_{l}\right)=\zeta\left(Q_{l}\right) \text {. }
$$

D'autre part, on a, d'après le lemme du $\mathrm{n}^{0}$ précédent (et d'ailleurs d'après ce que nous avons remarqué),

$$
\begin{gathered}
\zeta_{i}\left(P_{j}\right) \neq \zeta_{i}\left(P_{k}\right) \quad(j \neq k ; i=1,2), \text { et } \\
\zeta_{1}\left(P_{j}\right) \neq \zeta_{2}\left(P_{k}\right) \quad(j \neq l \text { ou } k \neq l) .
\end{gathered}
$$

De là, il résulte aussitôt que, parmi des valeurs de $\zeta_{1}(P)$ et de $\zeta_{2}(P)$ prises aux points $P_{1}, \cdots, P_{\nu}$ situés sur un point $\underline{P}$ de $\underline{T}-\underline{S}$, deux et seulement deux valeurs prises en un seul point $P_{l}$ sont égales:

$$
\zeta_{1}\left(P_{l}\right)=\zeta_{2}\left(P_{l}\right)
$$

et qu'on obtient $2 \nu-1$ valeurs mutuellement distinctes

$$
\zeta_{1}\left(P_{j}\right) \quad(j=1, \cdots, \nu) \text { et } \zeta_{2}\left(P_{k}\right) \quad(k=1, \cdots, \nu ; k \neq l) .
$$

De plus, puisqu'on a $\alpha_{1} \neq \alpha_{2}$, on a, au point $P_{l}$

$$
\frac{\partial \zeta_{1}}{\partial y}\left(P_{l}\right) \neq \frac{\partial \zeta_{2}}{\partial y}\left(P_{l}\right)
$$

les dérivées partielles étant prises par rapport aux coordonnées locales $((x), y)$ au voisinage (univalent) $d u$ point $P_{l}$ de $\mathscr{D}$. 
9. Considérons maintenant, à l'espace de $n+1$ variables complexes $((x), y, z)$, deux surfaces analytiques $\sum_{i}(i=1,2)$ :

$$
z=\zeta_{i}(P) \quad\left(P \in \mathscr{D}=\left(\mathscr{D}^{0}, Y\right)\right),
$$

$\zeta_{i}(P)$ étant les fonctions holomorphes sur $\mathscr{D}$, mentionnées au $\mathrm{n}^{0}$ précédent:

$$
\zeta_{i}(P)=\zeta(Q)+\alpha_{i}[y-\eta(Q)] \quad\left(P=(Q, y), Q \in \mathscr{D}^{0}\right) .
$$

$\Sigma_{i}$ passent évidemment par la variété donnée $T$ :

$$
y=\eta(Q), z=\zeta(Q) \quad\left(Q \in \mathscr{D}^{0}\right) .
$$

Soit $\Sigma_{0}$ la réunion de $\Sigma_{1}$ et $\Sigma_{2}$ :

$$
\Sigma_{0}=\Sigma_{1} \cup \Sigma_{2}
$$

$\Sigma_{0}$ admet $2 \nu$ feuillets comme un domaine intérieurement ramifié sur $\mathscr{D}=\left(\mathscr{D}^{0}, Y\right)$.

Désignons par $\hat{T}$ l'ensemble des points de $\Sigma_{0}$ situés sur $\underline{T}$ : $y=\eta(Q) ; \hat{T}$ est une variété de dimension $n-1$, et qui contient $T$. Soit $T^{*}$ la réunion des composantes de $\hat{T}$ qui n'appartiennent pas à $T$; on a

$$
\hat{T}=T \cup T^{*} \text {. }
$$

Pour voir l'allure de $\Sigma_{0}$ le long de $\hat{T}$, désignons par $S$ [resp. $S^{*}$ ] l'ensemble des points de $T$ [resp. $T^{*}$ ] situés sur les points de $\underline{S}=E \cap T ; S$ et $S^{*}$ sont également de dimension $n-2$.

D'après ce que nous avons vu au $\mathrm{n}^{0}$ précédent, on constate que :

a) par tout point de $T^{*}-S^{*}$ passe un feuillet (univalent) de $\Sigma_{0}$, et un seul;

b) par tout point de $T-S$ passent deux et seulement deux feuillets (univalents) de $\Sigma_{0}$ avec plans tangents (à $n$ dimensions) distincts (et qui ne sont pas parallèles à l'axe de z), c'est-à-dire, $T$ est une partie de la variété double ordinaire de $\Sigma_{0}$.

10. Enfin, en désignant par $Q_{1}, \cdots, Q_{\nu}$ les $\nu$ points de $\mathscr{D}^{0}$ situés sur un point $\underline{Q}=(x)$ de $\underline{D}^{0}-E^{0}\left(E^{0} \supset \underline{\sigma}^{0}\right)$, formons le produit

$$
\Phi((x), y)=\prod_{j=1}^{\nu}\left[y-\gamma\left(Q_{j}\right)\right]
$$


$\Phi((x), y)$ est un pseudo-polynôme de degré $\nu$ en $y$, à coefficients holomorphes dans $\underline{D}^{0}$. La surface $\underline{T}: y=\eta(Q)$ est exprimée par l'équation

$$
\Phi=0 .
$$

De même, si on désigne par $P_{1}, \cdots, P_{\nu}$ les $\nu$ points de $\mathscr{D}=\left(\mathscr{D}^{0}\right.$, $Y$ ) situés sur un point $\underline{P}=((x), y)$ de $\underline{D}-E$, chacun des produits

$$
\Psi_{i}((x), y, z)=\prod_{j=1}^{\nu}\left[z-\zeta_{i}\left(P_{j}\right)\right] \quad(i=1,2)
$$

est un pseudo-polynôme de degré $\nu$ en $z$, à coefficients holomorphes dans $\underline{\mathscr{D}}=\left(\underline{D}^{0}, Y\right)$. Chaque surface $\sum_{i}$ est exprimée par l'équation

$$
\Psi_{i}=0 \quad(i=1,2) .
$$

Posons

$$
\Psi=\Psi_{1} \Psi_{2} ;
$$

$\Psi$ est un pseudo-polynôme de degré $2 \nu$ en $z$, à coefficients holomorphes dans $\mathscr{D}$. La surface $\Sigma_{0}=\Sigma_{1} \cup \Sigma_{2}$ est exprimée par l'équation

$$
\Psi=0,
$$

et $\hat{T}$ par les deux équations

$$
\Phi=0, \quad \Psi=0 .
$$

D'après ce que nous avons vu au $n^{0} 8$ (ou au $n^{0}$ précédent), il est évident que $\Psi$ n'a pas de facteur multiple en aucun point de $\Delta=(\mathscr{D}, Z)$. Donc, d'après le théorème 2 , il existe un nombre complexe $c$ tel que:

$1^{\circ}$ l'équation

$$
\Psi+c \Phi^{3}=0
$$

définisse une surface analytique (à $n$ dimensions) $\Sigma$ dans $\Delta, \Psi$ $+c \Phi^{3}$ étant un pseudo-polynôme de degré $2 \nu$ en $z$, à coefficients holomorphes dans $\mathscr{D}$, et son ordre de zéros étant premier; et que $2^{\circ}$ la variété singulière $\tau$ de $\Sigma$ soit identique à l'intersection 
de la surface $(\underline{T}, Z): \Phi=0$ et de la variété singulière $\tau_{0}$ de $\Sigma_{0}$ : $\Psi=0$, c'est-à-dire, qu'on ait

$$
\tau=\tau_{0} \cap \hat{T} .
$$

Mais, d'après ce que nous avons vu au $\mathrm{n}^{0}$ précédent, on a

$$
\tau=\tau_{0} \cap \hat{T}=\left(\tau_{0} \cap T\right) \cup\left(\tau_{0} \cap T^{*}\right) \subset T \cup S^{*},
$$

$T$ étant la variété donnée qui est une partie de la variété double ordinaire de $\Sigma_{0}$, et $S^{*}\left(\subset T^{*}\right)$ étant de dimension au plus $n-2$.

Le théorème 2 , appliqué au cas où $p=3, q=2$ et au cas où $p=3, q=1$, montre que $T$ est encore la variété double ordinaire de $\Sigma$, et que $\Sigma$ admet un seul feuillet (univalent) en tout point de $T^{*}-S^{*}$. Donc, $\Sigma$ est la surface voulue, de type $(K)$, et le théorème 3 est complètement démontré.

11. Soit $\mathscr{D}^{0}$ un domaine univalent quelconque (fini ou non) dans l'espace de $n-1 \quad(n \geqq 1)^{15)}$ variables complexes $\left(x_{1}, \cdots, x_{n-1}\right)$, et soient $\mathscr{D}_{i}^{0}(i=1, \cdots, m) m$ domaines intérieurement ramifiés sur $\mathscr{D}^{0}$, à $\nu_{i}$ feuillets respectivement.

Désignons par $\mathscr{D}^{0}$ le domaine intérieurement ramifié sur $\mathscr{D}^{0}$, à $\nu=\sum_{i=1}^{m} \nu_{i}$ feuillets, obtenu comme la réunion de $\mathscr{D}_{1}^{0}, \cdots, \mathscr{D}_{m}^{0}$.

Soient $\eta(Q), \zeta(Q)\left(Q \in \mathscr{D}^{0}\right)$ deux fonctions holomorphes sur $\mathscr{D}^{0}$, $\eta(Q)$ étant supposée propre sur $\mathscr{D}^{0}$ et $\zeta(Q)$ quelconque. La restriction de $\eta(Q)$ [resp. $\zeta(Q)]$ sur chaque $\mathscr{D}_{i}^{0}$ sera noté $\eta_{i}\left(Q_{i}\right)$ [resp. $\left.\zeta_{i}\left(Q_{i}\right)\right]\left(Q_{i} \in \mathscr{D}_{i}^{0}\right)$.

Considérons $m$ surfaces analytiques (à $n-1$ dimensions) $T_{i}$ dans $\mathscr{D}=\left(\mathscr{D}^{0}, Y\right)$, et $m$ variétés analytiques (à $n-1$ dimensions) $T_{i}$ dans $\Delta=(\mathscr{D}, Z)$, définies respectivement par

$$
\begin{gathered}
y=\eta_{i}\left(Q_{i}\right) \quad\left(Q_{i} \in \mathscr{D}_{i}^{0}\right), \\
y=\eta_{i}\left(Q_{i}\right), \quad z=\zeta_{i}\left(Q_{i}\right) \quad\left(Q_{i} \in \mathscr{D}_{i}^{0}\right) .
\end{gathered}
$$

Par une démonstration pareille à celle du théorème 3 , on a le théorème suivant:

Théorème 3 bis. ${ }^{15)}$ Etant donnés $m$ entiers positifs $q_{i}(i=1$,

15) Voir la note 13) du bas de la page 292. 
$\cdots, m)$, il existe dans $\Delta$ une surface analytique (à $n$ dimensions) $\sum$, admettant chaque $T_{i}$ pour sa variété multiple ordinaire d'ordre $q_{i}(i=1, \cdots, m)$, sans aucune variété singulière de dimension $n-1$ en dehors de $T=\bigcup_{i=1}^{m} T_{i}$.

En outre, on peut construire $\Sigma$ de manière qu'elle admette $\nu^{*}=\sum_{i=1}^{m} q_{i} \nu_{i}$ feuillets sur $\mathcal{D}$, et qu'elle n'admette aucune singularité en dehors de $\hat{T}=\pi^{-1}(\pi(T))$, $\pi$ désignant l'opération de projection de $\sum \operatorname{sur} \mathscr{D}$.

\section{BIBLIOGRAPHIE}

[1] K. Oka, Sur les fonctions analytiques de plusieurs variables. VIII-Lemme fondamental, J. Math Soc. Japan 3 (1951), p. 204 214 et p. 259 278.

[2] H. Onishi, Quelques propriétés locales des domaines intérieurement ramifiés, J. Math. Kyoto Univ. 3 (1963 64), p. 319 346.

[3] H. Onishi, Quelques propriétés locales des domaines intérieurement ramifiés.II, J. Math. Kyoto Univ. 4 (1964), p.191 205. 